\title{
Confined Nystatin Polyenes in Nanopore Induce Biologic Ionic Selectivity
}

\author{
Khaoula Boukari, ${ }^{1,2}$ Guillaume Paris, ${ }^{1}$ Tijani Gharbi, ${ }^{1}$ Sébastien Balme, ${ }^{3}$ \\ Jean-Marc Janot, ${ }^{3}$ and Fabien Picaud ${ }^{1}$ \\ ${ }^{1}$ Laboratoire de Nanomédecine, Imagerie et Thérapeutique, EA 4662, Université de Bourgogne-Franche-Comté, \\ Centre Hospitalier Universitaire de Besançon, 16 route de Gray, 25030 Besançon Cedex, France \\ ${ }^{2}$ CINaM, CNRS UMR 7325, Aix-Marseille University, Campus de Luminy, 13288 Marseille Cedex 9, France \\ ${ }^{3}$ Institut Européen des Membranes, ENSCM, CNRS UMR 5635, Université de Montpellier, Place Eugène Bataillon, \\ 34095 Montpellier Cedex 5, France
}

Correspondence should be addressed to Fabien Picaud; fabien.picaud@univ-fcomte.fr

Received 29 March 2016; Accepted 22 May 2016

Academic Editor: David Cornu

Copyright ( $(2016$ Khaoula Boukari et al. This is an open access article distributed under the Creative Commons Attribution License, which permits unrestricted use, distribution, and reproduction in any medium, provided the original work is properly cited.

\begin{abstract}
Antifungal polyenes such as nystatin (or amphotericin B) molecules play an important role in regulating ions permeability through membrane cell. The creation of self-assembled nanopores into the fungal lipid membranes permits the leakage and the selectivity of ions (i.e., blockage of divalent cations) that cause the cell death. These abilities are thus of first interest to promote new biomimetic membranes with improved ionic properties. In the present work, we will use molecular dynamic simulations to interpret recent experimental data that showed the transfer of the nystatin action inside artificial nanopore in terms of ion permeability and selectivity. We will demonstrate that nystatin polyenes can be stabilized in a hydrophobic carbon nanotube, even at high concentration. The high potential interaction between the polyenes and the hydrophobic pore wall ensures the apparition of a hole inside the biomimetic nanopore that changes its intrinsic properties. The probability ratios of cation versus anion show interesting reproducibility of experimental measurements and, to a certain extent, opened the way for transferring biological properties in synthetic membranes.
\end{abstract}

\section{Introduction}

Nystatin (NYS) is a membrane-active polyene produced by Streptomyces noursei strains [1]. These antifungal molecules are amphiphilic $[2,3]$. They are composed of a polar head, a hydrophilic chain, and a hydrophobic chain. This amphiphilic character leads to their self-assembly in biological membranes. Indeed, polyenes can incorporate inside the lipid membrane and form a barrel [4] where all hydrophobic chains are turned on the external part of the barrel and face the lipids, while the hydrophilic chains form the inner part of the channel $[3,5]$. This configuration allows the barrel intercalation inside the lipid membrane (depending on the sterol molecules [6]) and plays an important role in the ion permeability and selectivity (especially to monovalent ions $[2,5]$ ). The mechanism responsible for the barrel formation is still under discussion but may follow the diagram illustrated in Figure 1. However, its ionic properties are involved in the antifungal action of the polyenes $[7,8]$.

Solid state nanopores appear now as excellent tools to mimic biological channel properties because of their combined well defined geometries (shape and dimensions) and mechanical robustness. But controlling the inner nanopore diameter is still very challenging since it necessitates controlling diameter range from subnanometers' to hundreds of nanometers' scale, depending on the applications. As already mentioned, solid state nanopores do not exhibit selectivity or activity without functionalization. Both the stability and the property of the solid state nanopores are highly dependent on the experimental conditions of formation (e.g., track etched nanopore) as well as used material. For several years, some applications were developed using solid state nanopores such 

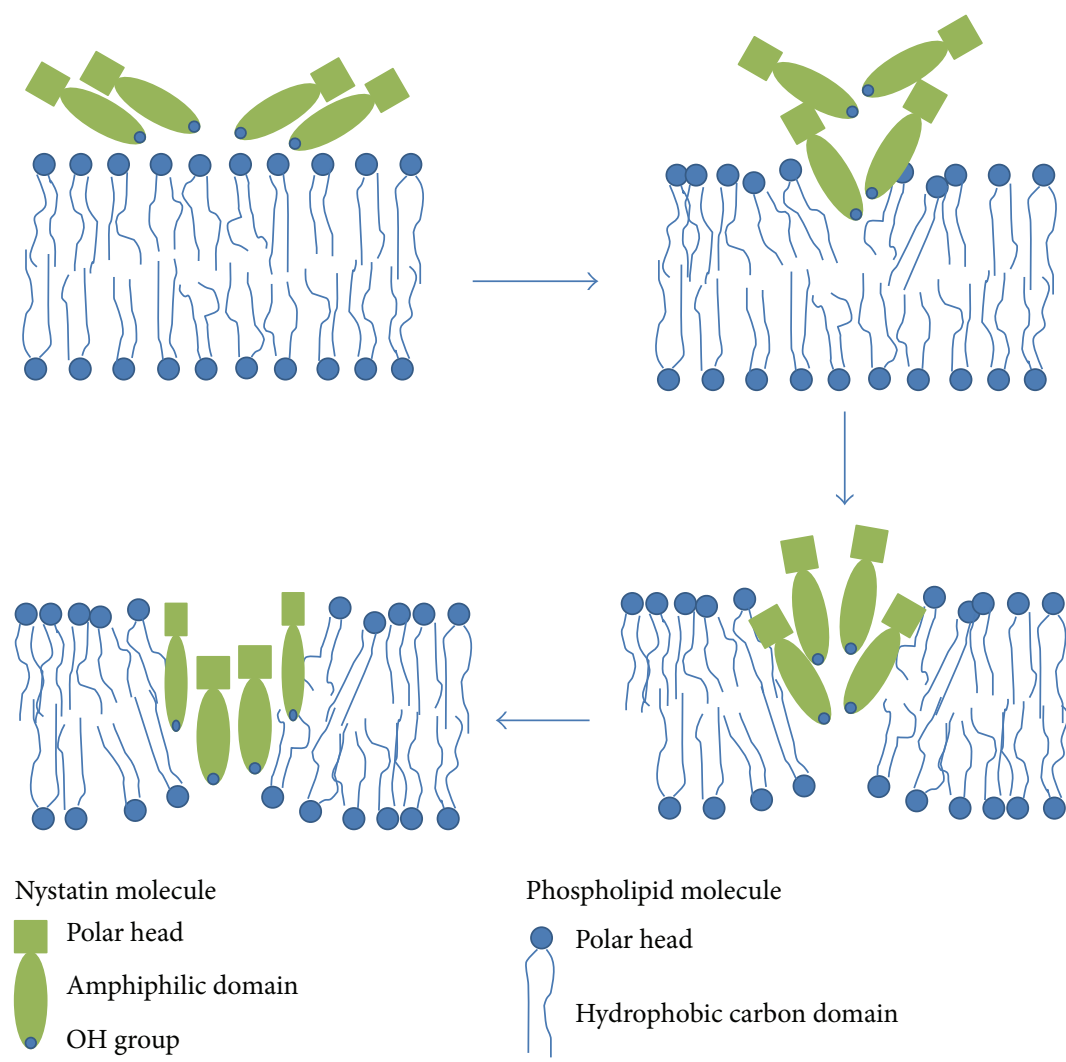

FIgURE 1: Schematic formation of the nystatin pore inside lipid membrane.

as DNA sensing [9], control of the molecular transport, or fabrication of performing nanofluidic devices [10-13]. Some precise chemical treatments allow now controlling the diameter range of nanopores $[14,15]$ and the properties [16]. However, if we want to combine the high selectivity of biological channel and the robustness of solid state nanopore, one route is the development of hybrid biological/solid state nanopores. This proof of concept was done with $\alpha$-hemolysin for DNA sensing application $[14,17]$ and with gramicidin A for higher ionic permeability and selectivity devices.

The transfer of the biological properties of polyene to artificial membranes which could thus exhibit the same behavior as in the cell could be envisaged but still remains an important challenge. This transfer could find applications in water desalination [18] or dialysis $[19,20]$ with low energy cost. Recently, Balme et al. demonstrated experimentally that insertion of NYS polyenes inside solid state nanopore leads to very interesting ionic selective properties. We will try here to understand using molecular dynamic simulations the formation and the properties of such experimental systems [20,21]. After a short description of the simulation method, we will present the progressive filling of the nanopore and the consequence to the properties of the polyenes in terms of geometry and energy. Then, we will analyze conductance of the hybrid nanopore and the role of the dipole moment, created after the polyenes incorporation in the ionic selectivity.

\section{Computational Method and Model}

The model for nystatin (NYS) was carried out using the 3D structure of the DB00681 model in the DrugBank database (Figure 2). To obtain the missing potential parameters, we have followed the protocol described by Norrby and Brandt [22] by constructing the Hessian matrix for further use in the force field parametrization. This matrix was obtained via $a b$ initio quantum calculations using Gaussian 09 package software [23]. In order to check the stability of polyene, nystatin was equilibrated for $2 \mathrm{nS}$ in a water box of $40 \times 30 \times 30 \AA^{3}$ (Figure 2). Then, NYS was transferred into a solid nanopore modeled by a carbon nanotube and denoted by CNT. Its diameter was chosen to be sufficient to be compared with experiments $[20,21]$, that is, $7 \mathrm{~nm}$, while its length was equal to $5 \mathrm{~nm}$. The nanopore was filled progressively by 50 polyenes, water, and $\mathrm{K}^{+}$and $\mathrm{Cl}^{-}$ions at $0.1 \mathrm{M}$ concentration. The final system contained 43702 atoms in a periodic box of $80 \times 75 \times$ $80 \AA^{3}$ with 10914 water molecules and $28 \mathrm{KCl}$ ions in order to conserve the neutrality of the system.

Molecular Dynamic (MD) simulations and minimization were performed using NAMD $2.7 \mathrm{~b} 2$ package [24]. A temperature of $300 \mathrm{~K}$ (Langevin dynamics) and a pressure of 1 atm (Langevin piston) of the system were kept constant during simulation. The long range electrostatic forces were evaluated by using the classical particle mesh Ewald (PME) 


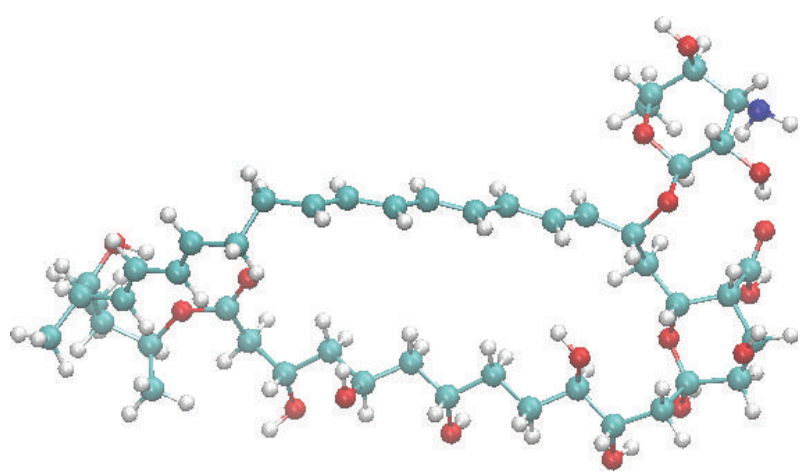

FIGURE 2: Structure of nystatin polyene.

method with a grid spacing of 1.2 and a fourth-order spline interpolation. The integration time step was equal to $1 \mathrm{fs}$.

To calculate the conductance in the hybrid membrane, electric field was imposed to the equilibrated system. The constant increase of the electric field value assures a voltage ramp which allows deducting the resulting ionic current. To do this, $25 \mathrm{nS}$ of MD simulations was performed for each electric field. The analyses of the ionic displacements inside the hybrid nanopore were carefully done in order to calculate the ionic current [25] using

$$
I(t)=\frac{1}{L_{y} \Delta t} \sum_{i=1}^{N} q_{i}\left(y_{i}(t+\Delta t)-y_{i}(t)\right)
$$

where $\Delta t$ represents the time between each frame, $L_{y}$ is the pore length $(5 \mathrm{~nm})$, and $y_{i}$ is the absolute position of the ion with charge $q_{i}$ along the pore axis.

\section{Results and Discussion}

In order to create the hybrid nanopore, CNT was progressively filled with the nystatin molecules. To do this, ten NYS molecules were incorporated in the center of the nanopore and stabilized by MD simulations. Then, once completely relaxed, ten other polyenes were added and so on, until 50 NYS molecules. For all systems, we observed a total aggregation of the molecules around the inner surface of the nanotube. This behavior was already reported for gramicidin [26] and amphotericin [20] molecules. The strong interaction of the hydrophobic chain of the polyenes with the hydrophobic CNT wall is at the origin of this behavior. It allows the polyenes recovering at best similar biological conditions. The control of the stability of each progressive filling was checked by the Root Mean Square Deviation (RMSD) of the NYS structure. Ten other NYS molecules were added once the RMSD value becomes roughly constant.

Figure 3 presents the different RMSD superimposed as a function of the time simulation. The results show that after $120 \mathrm{nS}$ of simulation time the whole system is perfectly stabilized. The deformation of the NYS skeleton is limited due to its confinement in the solid nanopore.

The different systems, modeled with 10, 20, 30, 40, and 50 nystatin molecules, are reported in Figure 4 . As clearly shown

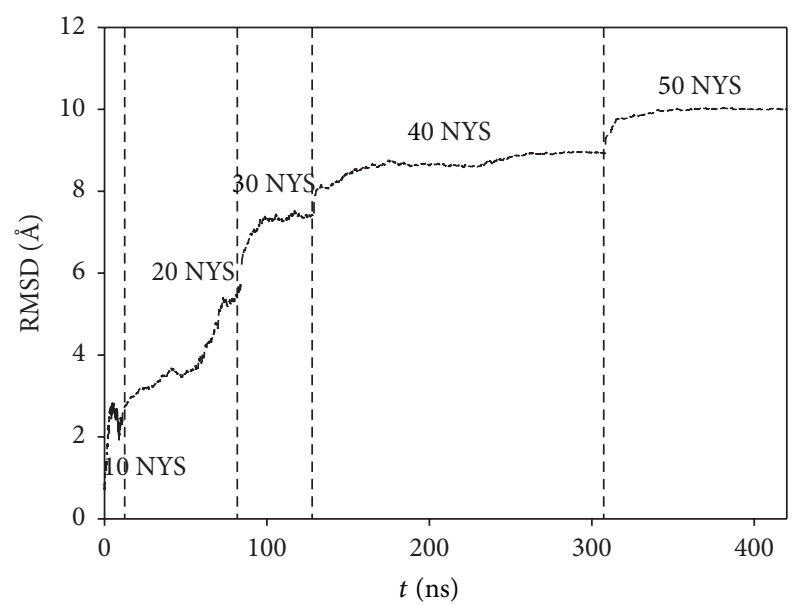

FIGURE 3: RMSD per added polyene for the progressive filling of the nanopore by the 50 nystatin molecules. For each concentration, the total RMSD was divided by 10 in order to calculate it as a function of the added NYS.

in these simulations snapshots, the polyenes tend to rapidly reach the pore wall and adsorb on it in order to optimize their interactions. After reaching a minimal concentration (40 NYS molecules were necessary to fully recover the pore wall), nystatin molecules were completely organized around the inner surface of the tube and a channel was formed in the tube center (see Figures 4(d) and 4(e) after insertion of 40 and 50 NYS molecules). It should be noted that the first 30 polyenes were more structurally perturbed (higher RMSD per NYS) than the following 20 ones, due to their adsorption on the CNT wall. Beyond 40 NYS molecules, the RMSD shows a slower increase since the latter NYS molecules are surrounded by other polyenes instead of CNT rigid wall. The snapshots showing the successive molecular arrangements during the simulation demonstrate the formation of a channel in the nanopore that could allow the ion diffusion. The approximate size of the different channels could be estimated using geometric arguments. To do this, we have divided the length of the pore in small portion of length $0.1 \mathrm{~nm}$. The minimal distance between each atom of faced NYS was then calculated to determine the diameter of the pore for the last two systems. The minimum value obtained, when 40 or 50 NYS molecules were confined, oscillated between $1.43 \mathrm{~nm}$ and $0.79 \mathrm{~nm}$. These values are significantly smaller than those of the CNT nanopore one $(7 \mathrm{~nm})$. The important decrease of the pore size, due to the presence of NYS molecules, could naturally affect the ions behavior through the application of an electric field. However, while confined in conditions far from biologic ones, the NYS molecules self-organize in the CNT to create a big channel. This behavior is similar in the fungal cell and allows the leakage of ions. The analysis of the different energy contributions is necessary to really understand the role of each type of molecules in the NYS behavior.

Figure 5 shows the van der Waals and electrostatic interactions per NYS molecule as a function of the simulation time. As shown on this figure, the total energy of the system 


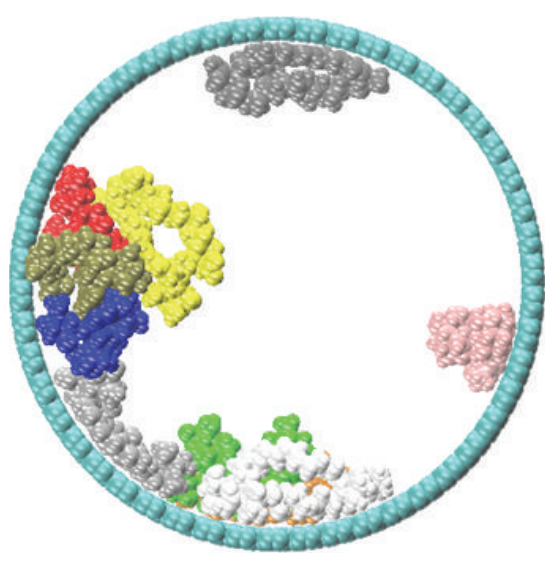

(a)

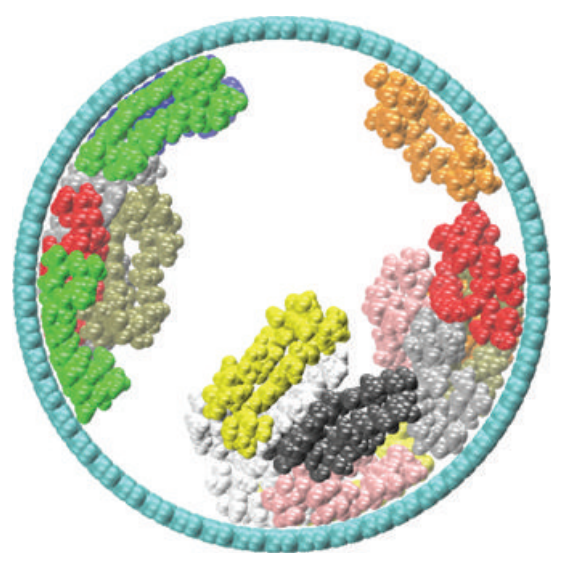

(b)

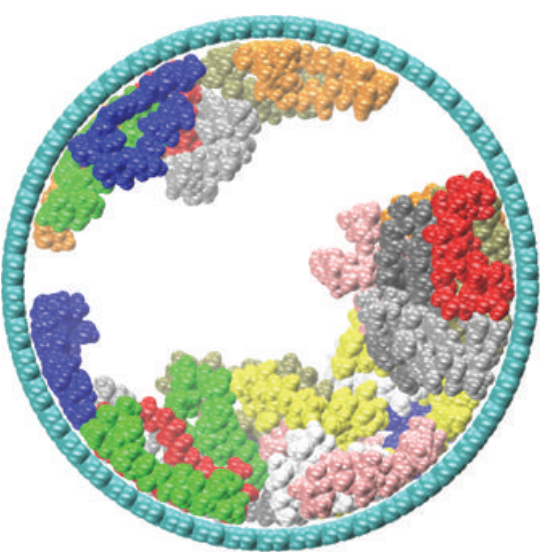

(c)

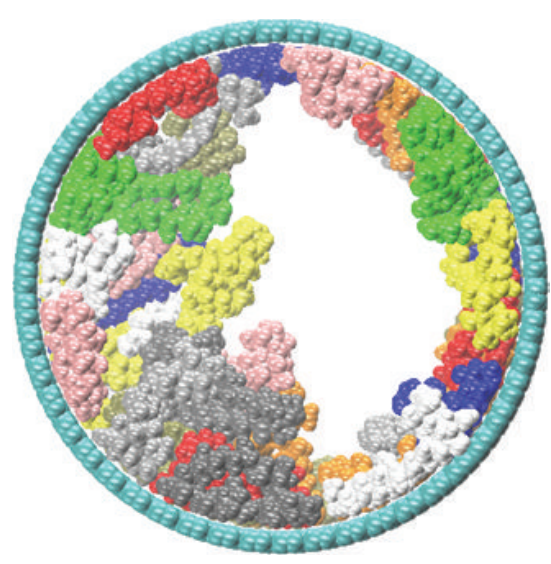

(d)

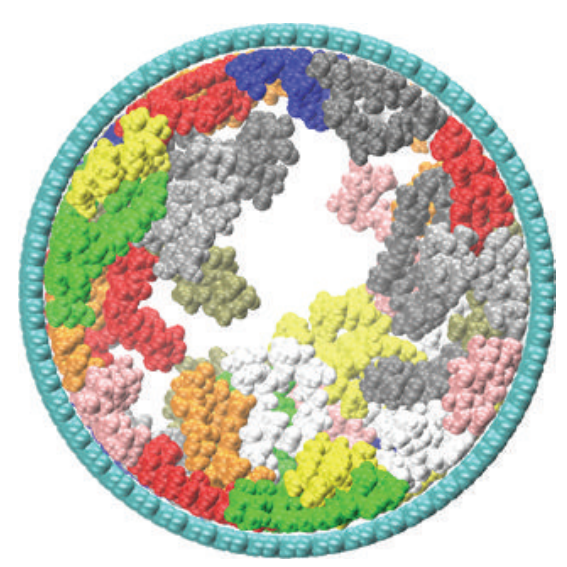

(e)

FIGURE 4: Snapshots of the simulation at (a) $30 \mathrm{nS}$ (10 nystatin molecules), (b) $81 \mathrm{nS}$ (20 nystatin molecules), (c) $129 \mathrm{nS}$ (30 nystatin molecules), (d) $310 \mathrm{nS}$ (40 nystatin molecules), and (e) $420 \mathrm{nS}$ (50 nystatin molecules). Carbon nanotube and nystatin polyenes are presented by cyan and different colors, respectively.

decreases until 20 encapsulated NYS molecules (from -273 to $-276 \mathrm{kcal} / \mathrm{mol})$. Then, the total energy per molecule increases slowly when the 20 following polyenes fill the CNT $(-270 \mathrm{kcal} / \mathrm{mol})$. At the final stage of the simulations $(50$ NYS molecules), the value of the energy is clearly higher $(-242 \mathrm{kcal} / \mathrm{mol})$, indicating a strong energy loss per molecule due to the 50 NYS molecules intense confinement. In order to explain this, we have separated in Figure 6 the different energy contributions responsible for the stability of the NYS molecules inside the nanopore. From the results, two main reasons can be given to explain the energy loss. First, the progressive insertion of polyenes inside the pore expels the water molecules in the reservoirs. This leads to the increase of the water/polyene energy contribution. Second, the completion of CNT inner surface was reached for 30 inserted molecules. The following ones can only interact with NYS wall which leads also to the slow increase of the CNT-NYS energy contribution. Nevertheless, the biomimetic system can be considered as stable since the lateral interaction between each NYS molecule should compensate the energy variation observed in the last system.
In order to confirm this, we have evaluated the average number of hydrogen bonds (Figure 7) formed between all polyenes during the last stage of the simulations (from $t=$ $340 \mathrm{nS}$ to $t=420 \mathrm{nS}$ ). A hydrogen bond was taken into account when the donor/acceptor distance was lower than $0.33 \mathrm{~nm}$ and the donor/hydrogen/acceptor angle was below $90^{\circ}$. The $\mathrm{H}$-bond average was computed as the total number of $\mathrm{H}$-bonds created in the system between NYS molecules during the simulation and divided by the frame number. An average of $14 \mathrm{H}$-bonds was found when NYS molecules were enclosed into the nanopore. This leads to an important cohesion of the molecules where they are confined and can be compared to value obtained for amphotericin when embedded inside membrane cell [6] (almost $10 \mathrm{H}$-bonds with sterol molecules). Of course, the number of $\mathrm{H}$-bonds evolves during the simulation, starting from some unities at the beginning of the filling to 14 near the completion of the nanopore (50 confined NYS polyenes). However, we can conclude that, progressively, the formation of this hydrogen bond lattice stabilizes the polyenes inside the hydrophobic nanopore and compensates the energy loss of the polyene with the other external components of the system. 


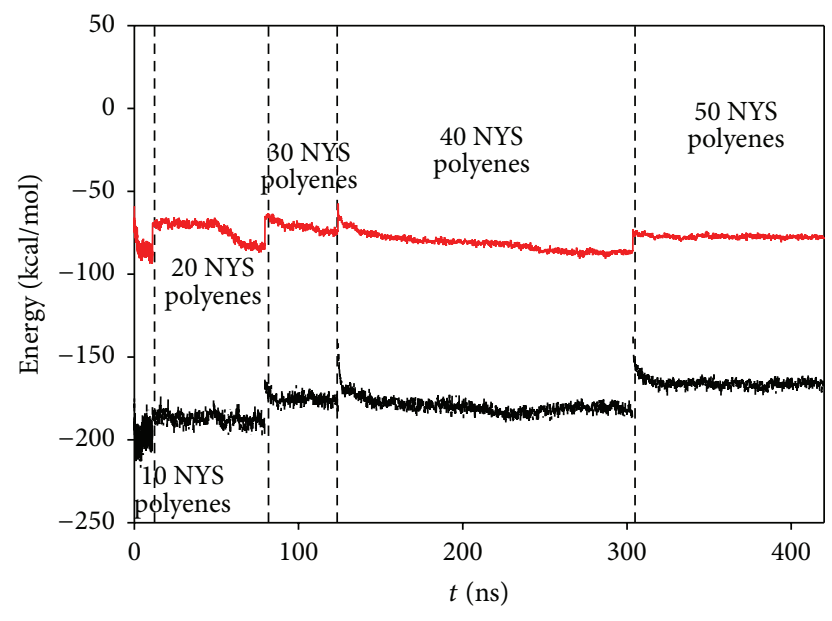

FIGURE 5: Total interaction energy of the polyenes with their neighbors (per nystatin polyene) as a function of time simulation (black curve for electrostatic interaction, red curve for van der Waals interaction).

In biological membrane NYS channel is well known to be permeable only to both monovalent cation and anion. This property should be studied in CNT in order to elucidate the role of the NYS confinement and self-assembly in the solid state nanopore on the permeation properties. To this end, membrane conductance $G_{\text {mem }}$ was determined through the current/voltage curve as a function of the NYS concentration. Figure 8 shows the different currents as a function of voltage obtained with the two last biomimetic systems (with 40 and $50 \mathrm{NYS}$ molecules) at $0.1 \mathrm{M}$ of $\mathrm{KCl}$.

As shown in Figure 8, $I=f(V)$ points are almost aligned. The linear regression of the curves can thus be used to determine the conductance for these two systems. When NYS occupied the inner part of the nanopore, the conductance was equal to $6.56 \mathrm{nS}$. In the second case, where $50 \mathrm{NYS}$ molecules filled the CNT, the conductance decreased to $3.44 \mathrm{nS}$. It should be noticed that the conductance calculated for the bare nanopore leads to a value of $168 \mathrm{nS}$. This conductance decrease can be directly assigned to the NYS confinement inside the membrane pore. Indeed, the inner diameter of the nanopore is first changed due to the polyene presence as well as the nature of the molecules that conducted ions (CNT versus NYS). When bare nanopore is considered, hydrophobic carbon wall limits the ions diffusion through the pore. On the other cases, ions face NYS polyenes when they diffuse. The consequent change of the membrane conductance is the combination of these two factors. Besides, by calculating the ratio between the conductance for 40 and that for 50 confined NYS polyenes, we retrieve almost the ratio of the channel radii. Note that we did not perform conductance calculations for different salts or different salt concentrations. However, the strong decrease of the membrane conductance in presence of confined polyenes is a sufficient proof of the functional behavior of our biomimetic nanopore. In addition, the recent experiments conducted on functional nanopore for the same concentration of $\mathrm{NaCl}$ or $\mathrm{KCl}$ did not show strong conductance differences between the two salts [20,21]. While the morphology of the organized polyenes inside the solid state nanopore was completely different to that observed in biologic lipid membrane, the resulting conductance behaves similarly with no difference between monovalent salts. This corroborates the partial biologic behavior of our functional nanopores. For divalent cations, conduction was also observed to a less extent while it was currently admitted that polyenes blocked the passage of divalent cations through a fungal cell. This can be due to the geometry adopted by the polyenes in CNT, which is far from its geometry in lipid cell.

In order to estimate the selectivity of the biomimetic nanopore demonstrated in $[20,21]$, we analyzed the dielectric dipole of the confined molecules as a function of time, omitting ions that could be present inside the nanopore during the calculations. This should help us to determine the probability of ion passage through the nanopore and, then, compare it to the permeation ratio measured in experiments.

The evolution of the dipole moment for two biomimetic systems (40 and 50 NYS molecules) shows important variations in the radial plane of the CNT. However, contrary to confined amphotericin B in similar CNT $[20,21]$, one of these components does not vanish during the simulation as shown in Figure 9. This can be interpreted as coming from the specific NYS organization inside the nanopore. While amphotericin B molecules were mainly organized homogeneously around the nanopore, nystatin polyenes create a channel which is quite twisted. This geometry can thus not compensate every component of the radial dipolar moment. The $(x, z)$ radial dipolar moment components vary around $-6 \pm 30 \mathrm{D},-60 \pm 30 \mathrm{D}$ (40 NYS molecules), and $-4 \pm 24 \mathrm{D}$, $-50 \pm 20 \mathrm{D}$ (50 NYS molecules), respectively. The component parallel to the nanopore axis fluctuates around a positive value of $60 \pm 30 \mathrm{D}$ and $40 \pm 30 \mathrm{D}$, respectively (see red arrow in Figure 9). This imposes an important orientation of the total dipolar moment along the nanopore axis (similar to a spin) which should impose an ionic selectivity along the pore.

The interaction between the dipolar moment along the pore axis and ion (cation or anion) inside the CNT nanopore can be calculated supposing that this ion, schematized by its own van der Waals radius, is in front of the CNT center. Following a Boltzmann distribution the probability ratio of finding $\mathrm{K}^{+}$and $\mathrm{Ca}^{2+}$ versus $\mathrm{Cl}^{-}$ions and finally $\mathrm{K}^{+}$versus $\mathrm{Na}^{+}$ions can be plotted as a function of time (Figures 10(a) and 10(b) for 40 and 50 confined NYS polyenes, resp.). From these results, it appears clearly that the probability of finding chlorine ions inside the channel is always higher compared to cations. This is due to a more favorable interaction with the dipolar moment imposed by the confined NYS polyenes. The divalent cations are poorly encouraged to diffuse through the NYS channel due to their higher positive charges (Figure 10, red lines). The value of $P_{\mathrm{K}} / P_{\mathrm{Na}}$ ratio (green curves) cannot be explained by the charge of the cations but only by the van der Waals radius between the two cations which involves a higher affinity to the membrane for potassium ions.

The change of the dipolar moment value between the two CNT fillings (40 and 50 NYS molecules) leads to low variation in the probability ratio. $P_{\mathrm{K}} / P_{\mathrm{Na}}$ ratio was modified from 1.14 to 1.08 while $P_{\mathrm{Ca}} / P_{\mathrm{Cl}}$ ratio changed from 0.005 


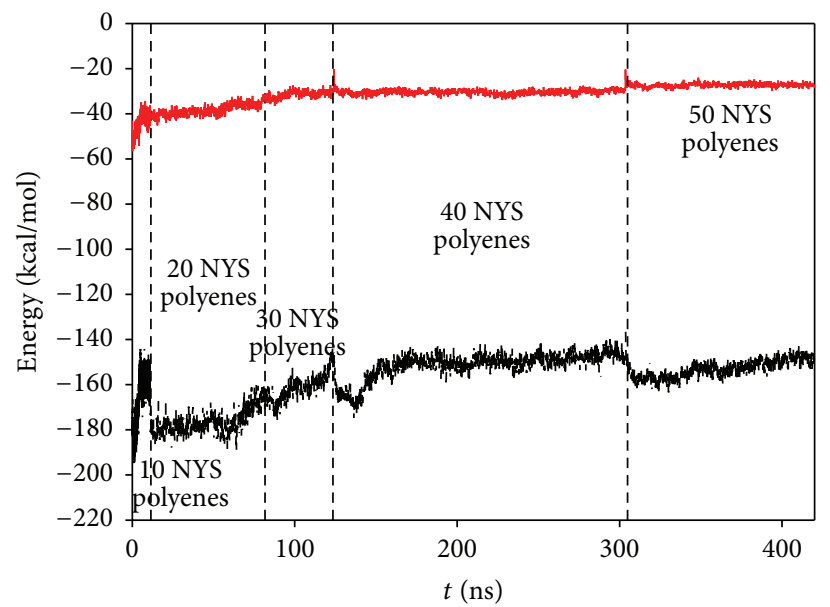

(a)

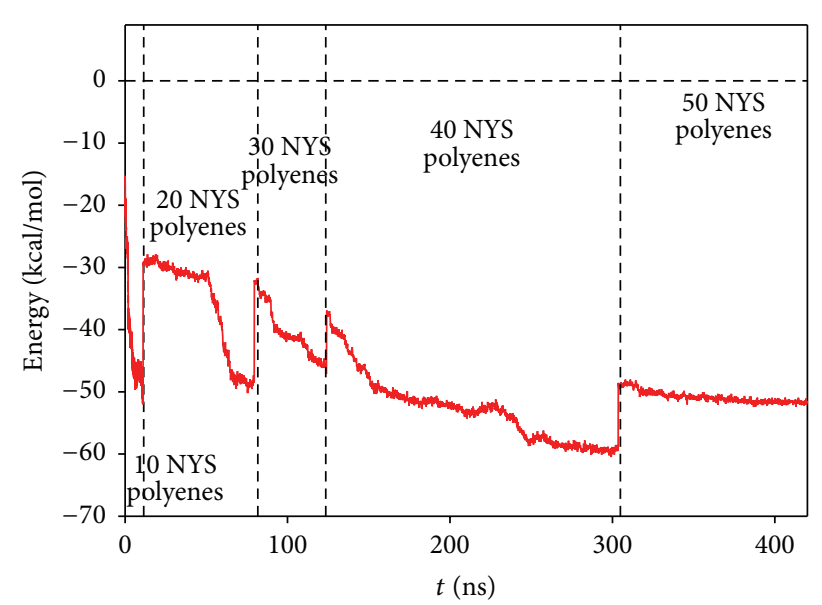

(b)

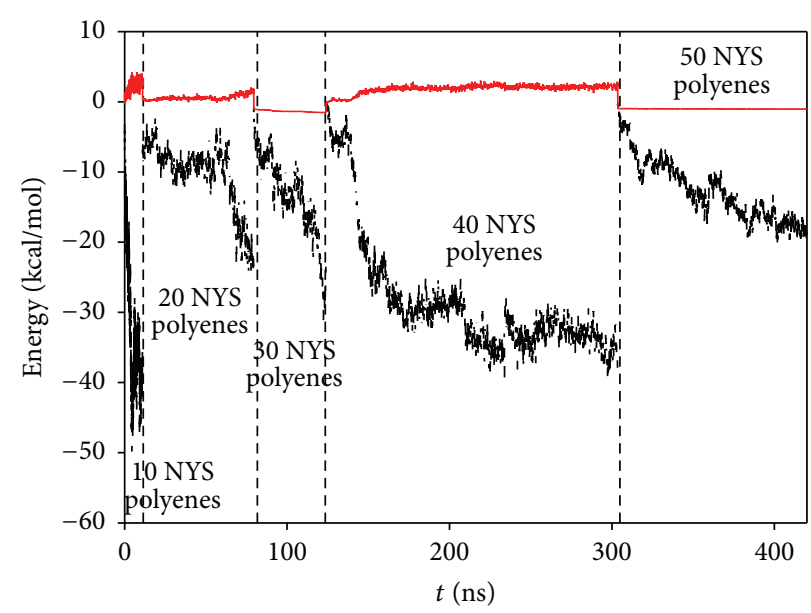

(c)

FIGURE 6: Energy contribution per nystatin polyene as a function of time simulation for (a) water molecules-NYS and (b) carbon atoms of the nanopore-NYS and (c) ions-NYS interactions.

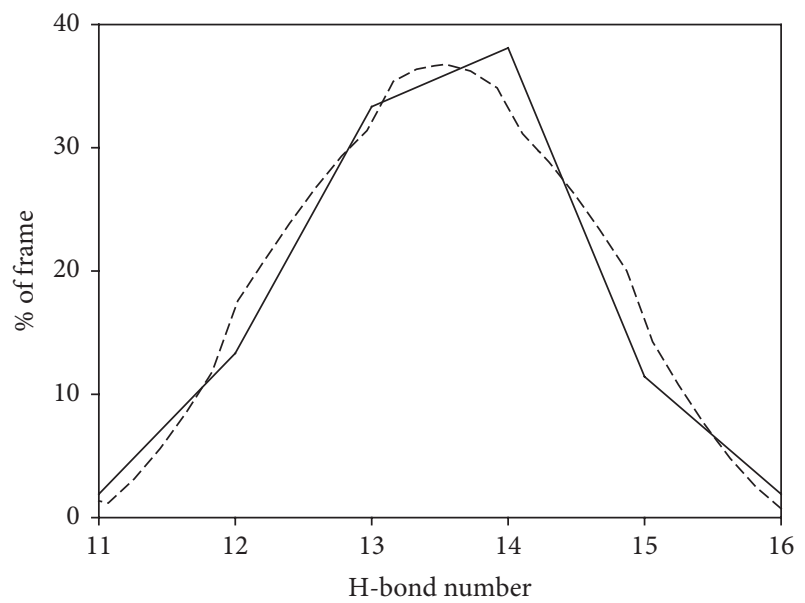

FIGURE 7: Average intermolecular hydrogen bonds number between all confined nystatin polyenes (solid line). The dashed line is an approximated Gaussian fitted curve of the simulation one. to 0.01 . The most important variation was observed for $P_{\mathrm{K}} / P_{\mathrm{Cl}}$ which increased from 0.12 to 0.3 . The biomimetic channels are thus extremely sensitive to monovalent ions and tend to promote anion diffusion compared to cation one. Nevertheless, the biologic properties observed in lipid membrane were not fully retrieved. Indeed, $P_{\mathrm{Ca}} / P_{\mathrm{Cl}}$ ratio can reach 0.01 which means that almost $1 \%$ of divalent cation could pass through the functional nanopore while $0 \%$ are awaited. The polyene disorder observed along the solid nanopore (fully different from the self-assembled channel observed in lipid membrane) and the lack of sterol molecules could explain the small differences observed between our biomimetic systems and the living cells.

However, our simulations agree well with the experiments recently published $[20,21]$. In these papers, Balme et al. demonstrated that it was possible to incorporate polyenes (amphotericin and nystatin) inside polycarbonate nanopore membrane with interesting ionic selective properties. While 


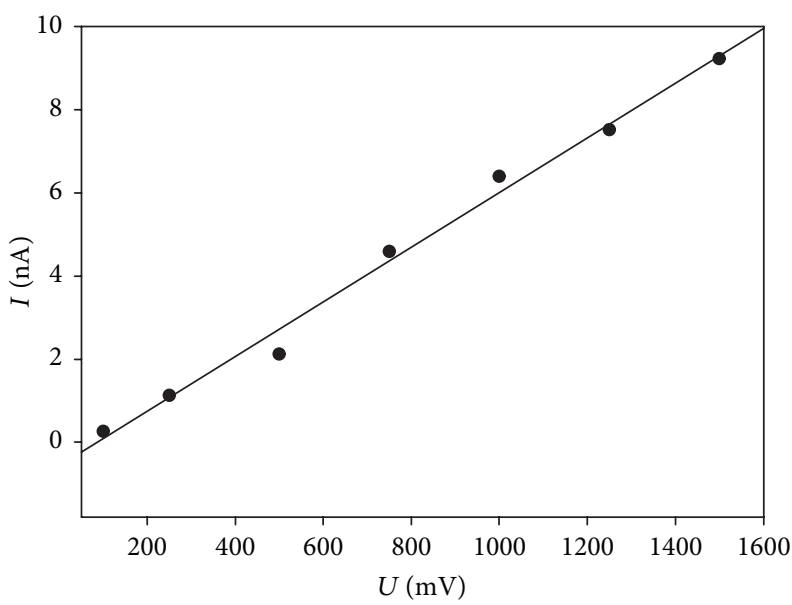

(a)

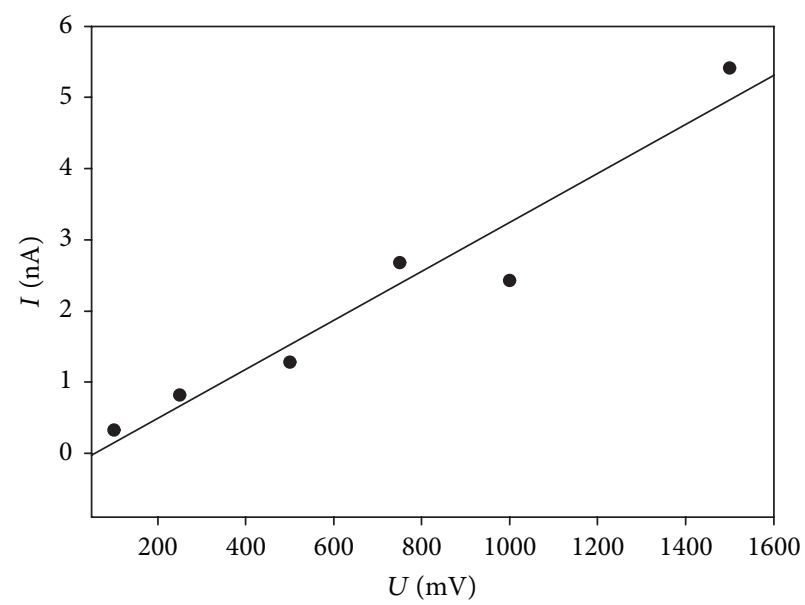

(b)

FIGURE 8: $I=f(V)$ curves obtained for systems with (a) 40 confined NYS polyenes and (b) 50 confined NYS polyenes. The biomimetic membrane conductance was equal to (a) $6.56 \mathrm{nS}$ and (b) $3.44 \mathrm{nS}$, respectively.

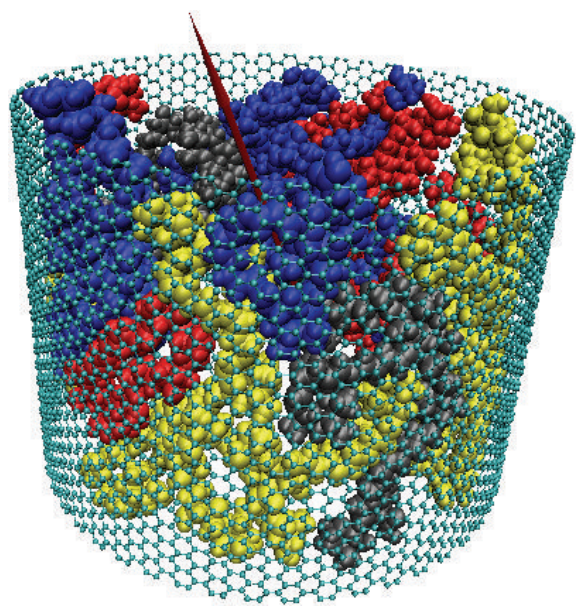

(a)

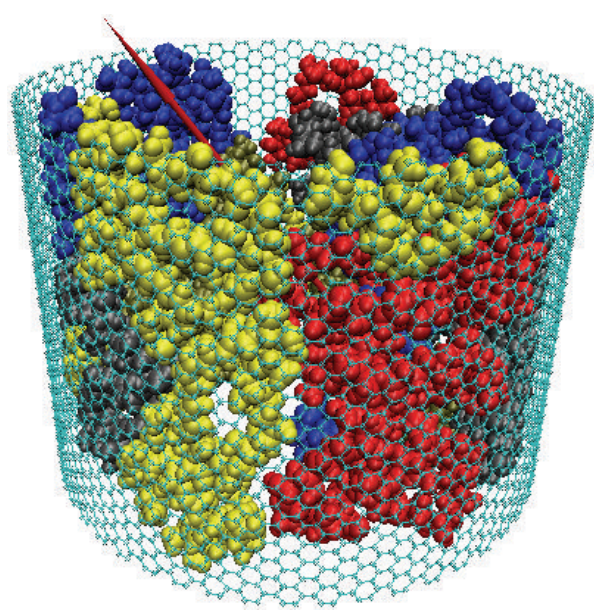

(b)

FIGURE 9: Dipolar moment of nystatin inside the nanopore for (a) 40 confined NYS polyenes and (b) 50 confined NYS polyenes. The red arrow presented the total dipolar moment. Carbon nanotube and nystatin polyenes are presented by cyan and different colors, respectively.

complete divalent cation selectivity was not retrieved (as in our simulations), these experiments showed that anions could cross the biomimetic membrane compared to cations. At salt concentration $0.1 \mathrm{M}$, the probability ratios $P_{\text {cat }} / P_{\mathrm{Cl}}$ obtained are 0.3 and 0.5 for $\mathrm{Na}^{+}$and $\mathrm{K}^{+}$, respectively, and 0.03 for divalent $\mathrm{Ca}^{2+} . P_{\mathrm{K}} / P_{\mathrm{Na}}$ ratio was found to be equal to 1.25 . Compared to our simulated systems, it appears that the experimental biomimetic channel is closer to the system simulated with 40 confined NYS polyenes, which corresponds to a channel of diameter $1.43 \mathrm{~nm}$. Indeed, in this case, the radius of the channel is larger and more symmetric than in the case where 50 NYS molecules were confined. This induced a more important directive dipolar moment that could help to increase selectivity.

\section{Conclusion}

Thanks to molecular dynamics simulations, we have conducted a theoretical study of the confinement of nystatin inside a carbon nanotube of $7 \mathrm{~nm}$ diameter. 50 NYS polyenes can fill the inner part of tube forming a hole in the center. The number of confined polyenes strongly influences the conductance of the biomimetic nanopore due to the modification of the ionic diffusing path. The resulting dipolar moment influences also the selectivity of the biomimetic membrane, with a strong preference for the anionic permeation compared to cationic one while divalent cations are almost blocked at the entry of the pore. Moreover, we have demonstrated here that the creation of the biomimetic channel reproduced selectivity 

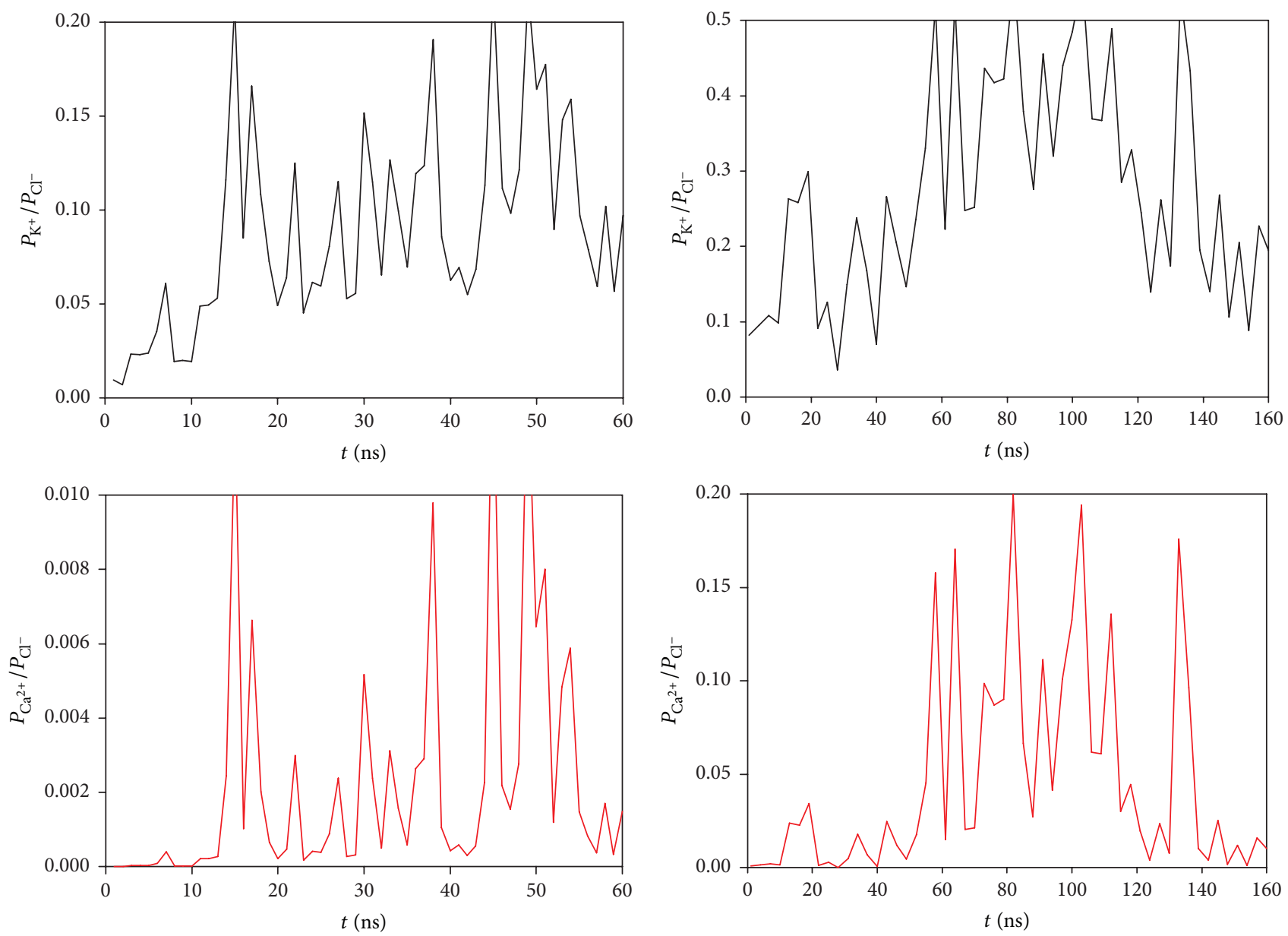

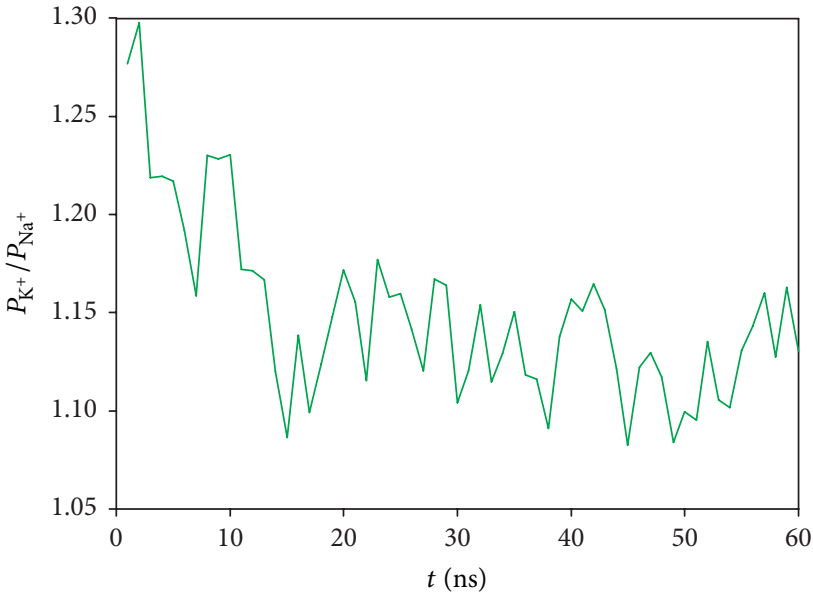

(a)

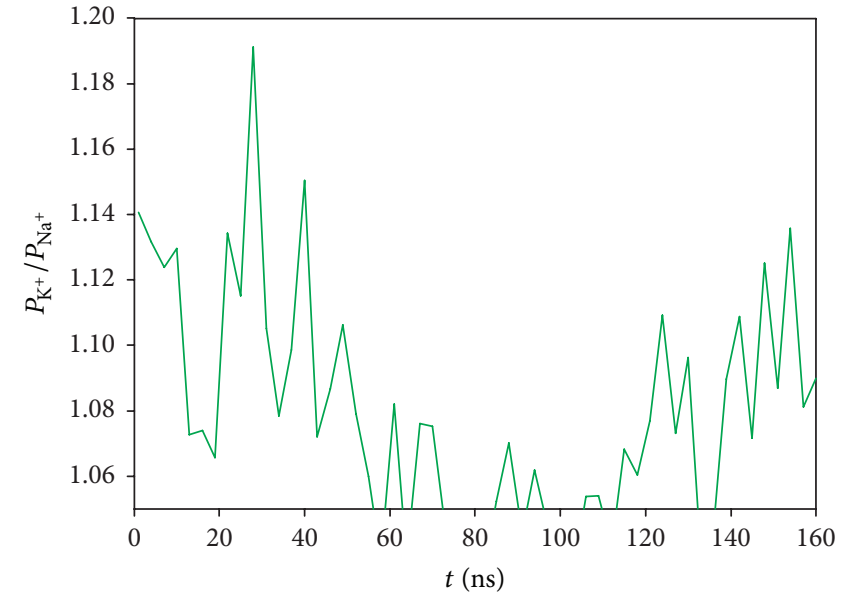

(b)

FIgURE 10: Ratio of the thermal equilibrium probability of $\mathrm{K}^{+}$(black line) and $\mathrm{Ca}^{2+}$ (red line) over $\mathrm{Cl}^{-}$and of $\mathrm{K}^{+}$over $\mathrm{Na}^{+}$(green line), as a function of time for (a) 40 confined NYS polyenes and (b) 50 confined NYS polyenes.

ratios close to those demonstrated experimentally [20, 21]. The functional nanopore exhibits interesting ionic properties that mimic partially the biologic behavior of NYS polyenes inside biological membrane. The organization of the polyenes inside the solid state nanopore is found to be completely different from that in lipid membrane. However, we succeed in modifying the solid state nanopore properties according to the confined molecules one. 


\section{Competing Interests}

The authors declare that there are no competing interests regarding the publication of this paper.

\section{Acknowledgments}

Computations have been performed on the supercomputer facilities of the Mésocentre de calcul de Franche-Comté. This work was supported by the French Research Program ANRBLANC, Project TRANSION (ANR-2012-BS08-0023).

\section{References}

[1] T. Brautaset, O. N. Sekurova, H. Sletta et al., "Biosynthesis of the polyene antifungal antibiotic nystatin in Streptomyces noursei ATCC 11455: analysis of the gene cluster and deduction of the biosynthetic pathway," Chemistry \& Biology, vol. 7, no. 6, pp. 395-403, 2000.

[2] N. Akaike and N. Harata, "Nystatin perforated patch recording and its applications to analyses of intracellular mechanisms," Japanese Journal of Physiology, vol. 44, no. 5, pp. 433-473, 1994.

[3] A. Cass, A. Finkelstein, and V. Krespi, "The ion permeability induced in thin lipid membranes by the polyene antibiotics nystatin and amphotericin B," The Journal of General Physiology, vol. 56, no. 1, pp. 100-124, 1970.

[4] J. Czub, A. Neumann, E. Borowski, and M. Baginski, "Influence of a lipid bilayer on the conformational behavior of amphotericin B derivatives-a molecular dynamics study," Biophysical Chemistry, vol. 141, no. 1, pp. 105-116, 2009.

[5] M. P. Borisova, R. A. Brutyan, and L. N. Ermishkin, "Mechanism of anion-cation selectivity of amphotericin B channels," The Journal of Membrane Biology, vol. 90, no. 1, pp. 13-20, 1986.

[6] K. Boukari, S. Balme, J. Janot, and F. Picaud, "Towards new insights in the sterol/amphotericin nanochannels formation: a molecular dynamic simulation study," The Journal of Membrane Biology, vol. 249, no. 3, pp. 261-270, 2016.

[7] J. Bolard, "How do the polyene macrolide antibiotics affect the cellular membrane properties?" Biochimica et Biophysica Acta (BBA)-Reviews on Biomembranes, vol. 864, no. 3-4, pp. 257304, 1986.

[8] J. Bolard, M. Seigneuret, and G. Boudet, "Interaction between phospholipid bilayer membranes and the polyene antibiotic amphotericin B. Lipid state and cholesterol content dependence," Biochimica et Biophysica Acta, vol. 599, no. 1, pp. 280293, 1980.

[9] G. F. Schneider and C. Dekker, "DNA sequencing with nanopores," Nature Biotechnology, vol. 30, no. 4, pp. 326-328, 2012.

[10] C. Y. Lee, W. Choi, J.-H. Han, and M. S. Strano, "Coherence resonance in a single-walled carbon nanotube ion channel," Science, vol. 329, no. 5997, pp. 1320-1324, 2010.

[11] P. Pang, J. He, J. H. Park, P. S. Krstić, and S. Lindsay, "Origin of giant ionic currents in carbon nanotube channels," ACS Nano, vol. 5, no. 9, pp. 7277-7283, 2011.

[12] A. Plecis, R. B. Schoch, and P. Renaud, "Ionic transport phenomena in nanofluidics: experimental and theoretical study of the exclusion-enrichment effect on a chip," Nano Letters, vol. 5, no. 6, pp. 1147-1155, 2005.

[13] R. B. Schoch, J. Han, and P. Renaud, "Transport phenomena in nanofluidics," Reviews of Modern Physics, vol. 80, no. 3, pp. 839$883,2008$.
[14] S. Cabello-Aguilar, S. Balme, A. A. Chaaya et al., "Slow translocation of polynucleotides and their discrimination by $\alpha$ hemolysin inside a single track-etched nanopore designed by atomic layer deposition," Nanoscale, vol. 5, no. 20, pp. 9582 9586, 2013.

[15] C. Marichy, M. Bechelany, and N. Pinna, "Atomic layer deposition of nanostructured materials for energy and environmental applications," Advanced Materials, vol. 24, no. 8, pp. 1017-1032, 2012.

[16] M. Lepoitevin, G. Nguyen, M. Bechelany, E. Balanzat, J.-M. Janot, and S. Balme, "Combining a sensor and a pH-gated nanopore based on an avidin-biotin system," Chemical Communications, vol. 51, no. 27, pp. 5994-5997, 2015.

[17] A. R. Hall, A. Scott, D. Rotem, K. K. Mehta, H. Bayley, and C. Dekker, "Hybrid pore formation by directed insertion of $\alpha$ haemolysin into solid-state nanopores," Nature Nanotechnology, vol. 5, no. 12, pp. 874-877, 2010.

[18] M. T. Myint, A. Ghassem, and N. Nirmalakhandan, "Low energy/cost desalination: Low dose and low mean ion resident time in concentrate stream of electro-dialysis reversal," Water Science and Technology, vol. 63, no. 9, pp. 1855-1863, 2011.

[19] J. Agar, "Solar-assisted haemodialysis," Clinical Journal of the American Society of Nephrology, vol. 7, no. 2, pp. 310-314, 2012.

[20] D. Thiele, S. Kraszewski, S. Balme, F. Picaud, J.-M. Janot, and P. Déjardin, "Structure and ionic selectivity of a hybrid polyene/ artificial polymer solid state membrane," Soft Matter, vol. 9, no. 3, pp. 684-691, 2013.

[21] S. Balme, D. Thiele, S. Kraszewski, F. Picaud, J.-M. Janot, and P. Déjardin, "Ionic selectivity of nystatin Al confined in nanoporous track-etched polymer membrane," IET Nanobiotechnology, vol. 8, no. 3, pp. 138-142, 2014.

[22] P.-O. Norrby and P. Brandt, "Deriving force field parameters for coordination complexes," Coordination Chemistry Reviews, vol. 212, no. 1, pp. 79-109, 2001.

[23] M. J. Frisch, G. W. Trucks, H. B. Schlegel et al., Gaussian 09, Gaussian, Wallingford, UK, 2009.

[24] J. C. Phillips, R. Braun, W. Wang et al., "Scalable molecular dynamics with NAMD," Journal of Computational Chemistry, vol. 26, no. 16, pp. 1781-1802, 2005.

[25] J. M. Xue, X. Q. Zou, Y. B. Xie, and Y. G. Wang, "Molecular dynamics simulations on the ionic current through charged nanopores," Journal of Physics D: Applied Physics, vol. 42, no. 10, Article ID 105308, 2009.

[26] S. Balme, J.-M. Janot, L. Berardo et al., "New bioinspired membrane made of a biological ion channel confined into the cylindrical nanopore of a solid-state polymer," Nano Letters, vol. 11, no. 2, pp. 712-716, 2011. 

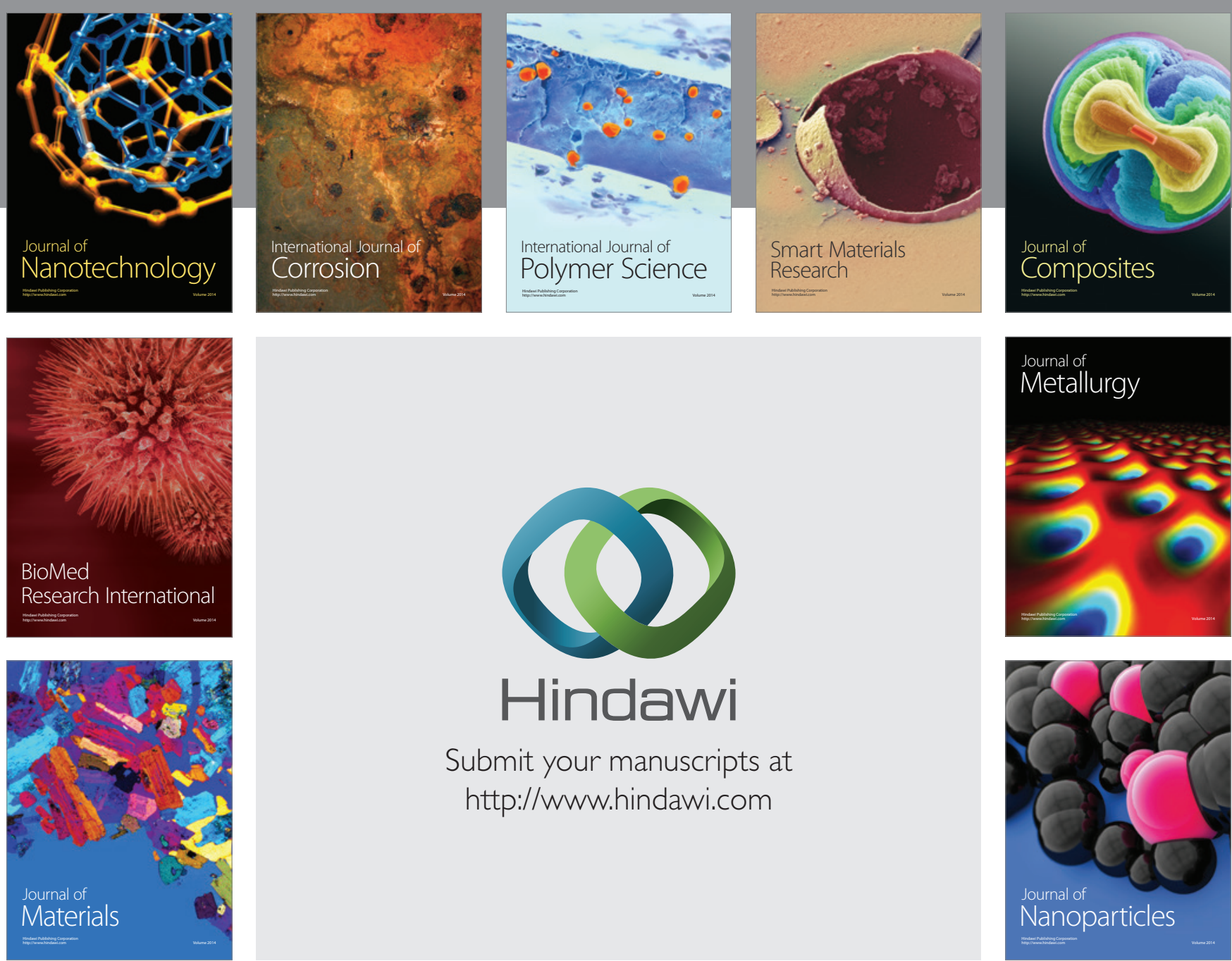

\section{Hindawi}

Submit your manuscripts at

http://www.hindawi.com

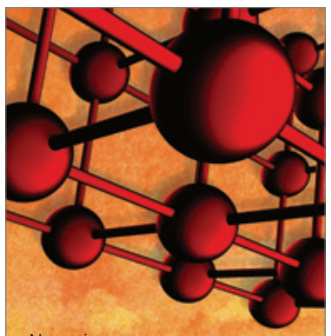

Materials Science and Engineering
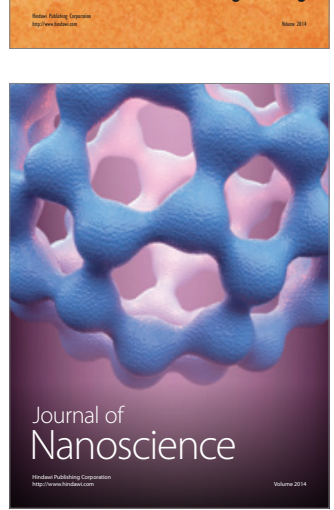
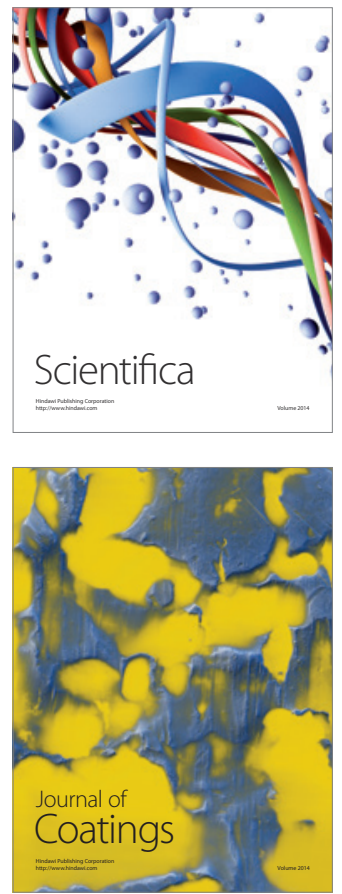
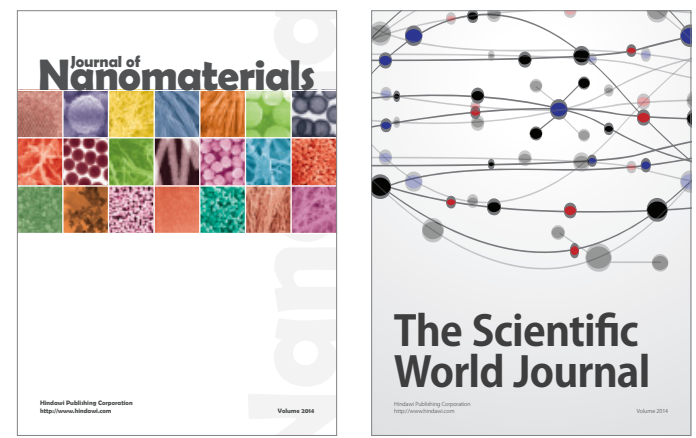

The Scientific World Journal
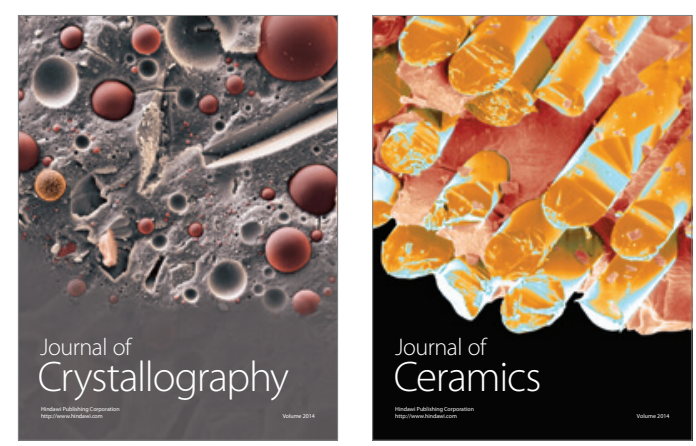
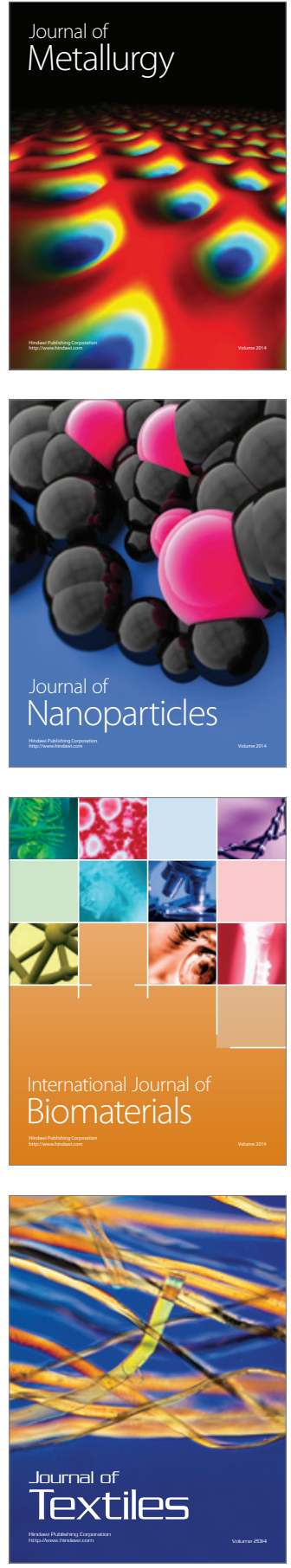